\title{
Phthalates, Pesticides, and Bisphenol-A Exposure and the Development of Nonoccupational Asthma and Allergies: How Valid Are the Links?
}

\author{
Eun Soo Kwak, ${ }^{1}$ Allan Just, ${ }^{2}$ Robin Whyatt, ${ }^{2}$ and Rachel L. Miller ${ }^{1,2 *}$ \\ ${ }^{I}$ Division of Pulmonary, Allergy, and Critical Care Medicine, Columbia University College of Physicians and Sur- \\ geons, USA \\ ${ }^{2}$ Mailman School of Public Health, Columbia University
}

\begin{abstract}
Phthalates, pesticides, and bisphenol-A (BPA) are three groups of chemicals, implicated in endocrine disruption and commonly found in the local environment, that have been implicated in the pathogenesis of asthma and allergies [1-3]. Multiple observational studies have demonstrated an association between exposure to phthalates and the development of asthma and allergies in humans. Associations with exposure to pesticides and BPA and the development of respiratory disease are less clear. However, recent evidence suggests that prenatal or early postnatal exposure to BPA may be deleterious to the developing immune system. Future cohort-driven epidemiological or translational research should focus on determining whether these ubiquitous chemicals contribute to the development of asthma and allergies in humans, and attempt to establish the routes and mechanisms by which they operate. Determining dose-response relationships will be important to establishing safe levels of these chemicals in the environment and in consumer products. Attempts to reduce exposures to chemicals such as phthalates, pesticides, and BPA may have environmental repercussions as well as public health impact for the developing child.
\end{abstract}

\section{INTRODUCTION}

The rise in asthma prevalence worldwide approximately parallels the rise in common use of several chemicals [4]. While asthma is a complex and heterogeneous disease associated with the inheritance of a genetic predisposition and exposure to many environmental agents, three groups of chemicals, associated with endocrine disruption and commonly found in the local environment, have been implicated recently in its pathogenesis. In this review, conducted through searches and study of recent literature listed in PubMed (www.PubMed.gov), we will discuss accumulating evidence supporting the associations between exposure to phthalates, pesticides, and bisphenol-A (BPA) and the development of asthma and allergies.

\section{PHTHALATES}

Phthalates are diesters of benzenedicarboxylic acid and are the most commonly used plasticizers today. Phthalates with the highest production volume are diisononyl phthalate (DiNP), diisodecyl phthalate (DiDP), and di-2-ethyl-hexyl phthalate (DEHP) [2, 5]. Phthalates are used as plasticizers to increase stability and flexibility, to prevent brittleness, as a solvent for fragrances, and as inert ingredients. Phthalates are found in vinyl floor tiles, carpet backing, paints, and wall materials. The majority of DEHP is used as a plasticizer in polyvinyl chloride (PVC) plastics, where it can be $40 \%$ of the finished product by weight [6]. Phthalates are commonly found in many other consumer products including shower curtains, adhesives, synthetic leather, toys, cosmetics, and medications. Human exposure to phthalates is evidenced by

*Address correspondence to this author at PH8 E, $610 \mathrm{~W} .168^{\text {th }} \mathrm{St}$, New York, NY, 10032, USA; Tel: 212-305-7759; Fax: 212-305-2277; E-mail: rlm14@columbia.edu presence of their metabolites in human urine and other biological samples [7-11]. Diet is believed to be the main source of DEHP exposure in the general population with higher concentrations found in high fat foods like dairy, poultry, and oils $[6,7,12]$. Ingestion of dust and mouthing of toys may be important additional sources of exposure for infants and toddlers $[6,7,9,12,13]$. Because they are not covalently bound into plastic when used as plasticizers, phthalates have been found to leach or migrate from PVC-containing items into air, dust, water, soils, and sediment [14].

Multiple studies have examined the association between the presence of PVC in the home flooring and walls, a presumed indicator of phthalate exposure, with the development of asthma and allergies in children. In a Finnish populationbased cross-sectional study of 2,568 children 1-7 years of age, the risks of wheezing, persistent phlegm, weekly nasal congestion and respiratory infections were related to the presence of plastic wall materials (presumably including PVC) at home [15]. In a Norwegian matched case-control study of 251 cases of bronchial obstruction and 251 controls, Jaakkola et al. found that the risk of bronchial obstruction was greater in the presence of known PVC in the floors [16]. Also, in a Swedish cross-sectional study of 10,851 children, the combination of water leakage and presence of PVC flooring was a strong determinant of doctor-diagnosed asthma and rhinitis compared with those children without leakage and PVC flooring [17]. In their follow-up study of cohort participants recruited 1.5 years later, 198 subjects with persistent allergic symptoms present both at baseline and on follow-up, as well as 202 controls, underwent medical examination and measurement of home dust concentrations of six phthalates (diethyl phthalate (DEP), diisobutyl phthalate (DIBP), di-n-butyl phthalate (DnBP), n-butyl benzyl phthalate (BBzP), diethyl phthalate (DEHP), and diisononyl phthalate (DINP)). Subjects with persistent allergic 
symptoms were more likely to have PVC flooring in the bedroom. Importantly, the median house dust concentrations of $\mathrm{BBzP}$ were higher in the bedrooms of subjects with rhinitis and eczema than in those of controls, and the median level of DEHP was greater among those with reports of physician diagnosis of asthma [18]. This study design was replicated in Bulgaria in 2004-2005 with 102 cases and 82 controls with higher DEHP in house dust from cases and a dose-response increase in the odds of being a case or reporting wheeze in the previous 12 months with increasing quartile of DEHP. In Bulgaria, BBzP levels were non-significantly higher in the homes of those reporting wheeze or eczema [19].

Toxicology studies, conducted both in vitro and in vivo animal models, have proposed various mechanisms by which phthalates may exert their effect on airway inflammation. For example, Nakamura et al. examined the effect of three dialkyl phthalates, di-n-butyl phthalate (DnBP), diisobutyl phthalate (DIBP) and di-2-ethyl-hexyl phthalate (DEHP), on antigen-induced degranulation of rat basophilic leukemia (RBL)-2H3 mast cells. Exposure to 50-500 $\mu \mathrm{m}$ DnBP, 50$500 \mu \mathrm{m}$ DIBP, and $500 \mu \mathrm{m}$ DEHP significantly potentiated antigen-induced beta-hexosaminidase release. It was concluded that some dialkyl phthalates increase antigen-induced degranulation in RBL-2H3 cells dependent on the increase of cytosolic calcium ion concentrations [20]. Glue et al., in one of the first studies to test potential proallergic adjuvant effects associated with phthalate exposure, found that incubation of the monocytic THP-1 cell line or peripheral blood mononuclear cells (PBMCs) with monophthalates induced virtually no changes in cytokine expression. However, ex vivo incubation of $\mathrm{PBMCs}$ collected from allergic individuals with mono-n-butyl phthalate (MnBP) tended to increase the level of interleukin [IL]-4 production [21]. In a subsequent study by the same group, rapid histamine release occurred when human PBMCs were incubated with DEHP or mono-(2-ethylhexyl) phthalate (MEHP), then stimulated with anti-immunoglobulin (Ig) E, N-formyl-methionylleucyl-phenylalanine (fMLP), calcium ionophore, or cat hair allergen extract. Exposure to the 8-carbon phthalates (DEHP, MEHP, MOP, and DOP) caused the strongest histamine release, whereas incubation with 4-, 9-, or 10-carbon phthalates (MBuP, DBuP, MINP, DINP, MIDP, and DIDP) caused no or low induction of histamine [22]. Jepsen et al. showed that the extent of adjuvancy provoked by individual phthalates was structure-related. After exposure to monophthalates, concentrations of the proinflammatory cytokines IL-6 and IL-8 were measured in the human epithelial cell line A549. The study demonstrated that some, but not all, monophthalates could induce a concentration-dependent increase in cytokine production. At higher concentrations, all phthalate doses suppressed cytokine production. Monophthalates with fewer than eight carbon atoms in their alkyl side chain were weak cytokine inducers, whereas monophthalates with eight or more carbon atoms in the alkyl side chain were more potent cytokine inducers. Cytokine suppressive effect of the monophthalates also increased with increasing alkyl side chain length [23].

In an early study in rats, Klimisch et al. exposed 9-weekold animals to DEHP aerosols 6 hours/day, 5 days/week, for 4 weeks with estimated doses of 230,11 and $2.3 \mathrm{mg} / \mathrm{kg} / \mathrm{day}$ for the males, and 360, 18 and $3.6 \mathrm{mg} / \mathrm{kg} /$ day for females. A statistically significant increase $(16 \%)$ in relative lung weights, accompanied by thickening of the alveolar septi, was found in the males exposed to the highest dose (230 $\mathrm{mg} / \mathrm{kg}$ ). All of these effects were reversed 8 weeks after termination of the aerosol [24]. Larsen et al. designed a series of studies to investigate whether a panel of monophthalates (i.e. the principal metabolites of phthalates) had adjuvant properties in vivo among mice sensitized to ovalbumin (OVA). While some immunosuppression was observed following exposure to high doses of MEHP, mono-n-octyl phthalate, mono-iso-nonyl phthalate and mono-isodecyl phthalate (MOP, MINP and MIDP) significant increases in proallergic $\mathrm{IgE}$ and $\mathrm{IgG}_{1}$ production occurred following exposure to MEHP (10 $\mu \mathrm{g} / \mathrm{ml}, \mathrm{IgE}), \mathrm{MOP}(100 \mu \mathrm{g} / \mathrm{ml}$, and 10 $\left.\mu \mathrm{g} / \mathrm{ml} \mathrm{IgG}_{1}\right)$ and MINP (100 $\left.\mu \mathrm{g} / \mathrm{ml}, \mathrm{IgE}\right)$ [25]. Next, Larsen et al. studied the adjuvant effects of di-n-butyl-, di-n-octyl-, di-iso-nonyl- and di-iso-decyl phthalates $(2-2000 \mu \mathrm{g} / \mathrm{ml})$ in a similar mouse model. Phthalates with 8 or 9 carbon atoms in the alkyl side chains induced greater $\operatorname{IgE}, \operatorname{IgG}_{1}$ production compared to phthalates with shorter or longer alkyl side chains [26].

Hansen et al. investigated whether exposure to MEHP induced adjuvant effects in a mouse inhalation model following chronic exposure to low dose OVA. Among mice exposed to aerosols of MEHP, OVA-specific $\operatorname{IgG}_{1}$, but not $\operatorname{IgE}$ or $\operatorname{IgG} 2_{\mathrm{a}}$, production was increased when compared to mice sensitized to OVA with adjuvant $\mathrm{AI}(\mathrm{OH})_{3}$ only. A dosedependent increase in eosinophils and lymphocytes also was observed in bronchoalveolar lavage fluid. Ex vivo cytokine secretion by cultures of draining lymph nodes also suggested a proallergic $\mathrm{T}$ helper (Th) 2 profile following exposure to MEHP [27].

Despite studies that have suggested an association between phthalate exposure and the development of asthma and allergy, one animal model showed a lack of such a relationship. Topical administration of undiluted DEHP, DINP, n-butyl benzyl phthalate (BBzP) and di-isohexyl phthalate (DIHP) to the flanks of mice $(50 \mu \mathrm{L} /$ flank) in the absence of allergen exposure, followed by low dose topical challenge to the ear $(25 \mu \mathrm{L} / \mathrm{ear})$ one week later, did not affect the levels of proallergic cytokine or IgE production [28].

Interestingly, a recent study examined the effect of maternal exposure to DEHP during fetal and/or neonatal periods on subsequent dust mite allergen-induced atopic dermatitis in the offspring. DEHP was administered intraperitoneally at a weekly dose $(0.8-100 \mu \mathrm{g})$ per dam during pregnancy and/or lactation. Eight-week-old male offspring were challenged with intradermal injection of mite allergen into their ears. Maternal exposure to DEHP $(100 \mu \mathrm{g})$ during lactation periods, but not during fetal periods, augmented the development of ear thickening and atopic dermatitis-like skin lesions at the site of allergen injection. Histological evaluation of the tissue showed greater infiltration of eosinophils, mast cell degranulation, and protein expression of eotaxin, consistent with DEHP-induced Th2-dominant allergic dermatitic responses. These effects were not replicated in female offspring, suggesting hormone-mediated processes in the observed phthalate-induced early postnatal effects on allergic immune responses [29]. 


\section{PESTICIDES}

Worldwide there continues to be widespread use of pesticides and herbicides in agriculture, as well as residential pesticide use. While it has been evident for a long time that occupational exposure to pesticides is associated with wheeze and other respiratory symptoms in agricultural workers [1, 30,31 , its association with similar symptoms among nonoccupationally exposed individuals, including children, has gained more recent attention.

For example, Salameh conducted a cross-sectional study on 4000 children from a randomly selected sample of 18 Lebanese public schools assessing the association of exposure to pesticides and reported chronic respiratory symptoms. Using questionnaire data, approximately $12 \%$ of children were reported to have a chronic respiratory disease, defined as recurrent cough, expectoration, wheezing with or without dyspnea. Exposure to pesticides was divided into residential, para-occupational and domestic. Residential exposure was characterized by residing in the proximity of a treated field. Domestic exposure was defined as any domestic use by a household member or gardener. Paraoccupational exposure was defined as occupational use of pesticides by one or the household members. After controlling for exposure to environmental tobacco smoke in the home, any (residential, domestic or paraoccupational) exposure to pesticides was associated with doctor-diagnosed asthma, chronic cough, chronic phlegm, recurrent wheezing, and ever wheezing [32].

Salam et al. conducted a retrospective, prevalence casecontrol study nested within the Children's Health Study [5] in order to test the hypothesis that environmental exposures in early childhood are associated with increased occurrences of early transient wheezing and/or early-onset persistent asthma. Of 4,244 children who were between 8 and 18 years old at the time of enrollment, 338 children with asthma before 5 years of age, and 570 non-asthmatic controls were matched for age, sex, community of residence and in utero exposure to maternal smoking. Telephone interviews were conducted with mothers to collect additional exposure and asthma histories. Reported exposure to wood or oil smoke, soot, exhaust, dust, herbicides and pesticides during the first year of life was significantly associated with the development of asthma by 5 years of age [5]. However, O'Sullivan et al. demonstrated that during the 1999 eradication program of the mosquito vector for West Nile virus in New York City, the spraying of insecticides (malathion, resmethrin) was not associated with an increased rate of emergency room visits or hospitalizations for asthma [33].

Multiple mechanisms have been proposed to explain the effects of exposure to pesticides and the development of asthma or allergy development. Most pesticides are small molecules and can exacerbate asthma and atopic dermatitis symptoms by Type-I and -IV hypersensitivity mechanisms [34]. Exposure to organophosphate and carbamate compounds may trigger respiratory symptoms through cholinesterase inhibition that may promote bronchoconstriction [1]. Fryer et al. developed a model of organophosphate-induced airway hyperreactivity in guinea pigs, which suggested that organophosphate-induced airway hyperreactivity results from effects of autoinhibitory M2 muscarinic receptors on the parasympathetic nerves in the lung, not from acetylcholinesterase inhibition or dysfunction of M3 muscarinic receptors in airway smooth muscle [35].

\section{BISPHENOL-A}

BPA is used in lacquers to coat food cans and water pipes, and it is also found in dental sealants and composites. Like phthalates, the main source of exposure is believed to be dietary but is not yet well understood. The use of drinking containers made with polycarbonate plastics has been associated with higher exposure [36, 37]. BPA is used in the manufacture of epoxy, polycarbonate plastics, vinyl and unsaturated polyester resins. It also has been used as an antioxidant, fungicide, and antimicrobial in cosmetics. More than a dozen studies using a variety of different analytical techniques have measured free, unconjugated BPA concentrations in human serum at levels ranging from $0.2-20 \mathrm{ng} / \mathrm{ml}$ serum [38].

BPA has been measured in human urine from several populations around the world, and the relatively high levels of BPA in the serum of pregnant women, umbilical cord blood, and fetal plasma indicate that BPA crosses the maternal-fetal barrier [39]. In fact, some of the highest levels of BPA reported in human tissue $(8.3 \mathrm{ng} / \mathrm{ml})$ occur in amniotic fluid between weeks 15-18 of pregnancy [40]. The latter finding is particularly concerning in light of studies demonstrating that maternal-fetal transmission of BPA, even in small doses, is especially deleterious. For example, Maffini et al. reported that perinatal exposure to BPA in mice resulted in morphological and functional alterations of the male and female genital tract and mammary glands that may predispose the tissue to earlier onset of disease, reduced fertility and mammary and prostate cancer [3]. The effects of postnatal exposure to BPA also are concerning. For example, Vandenberg et al. showed that in CD-1 mice exposed to BPA during gestation and lactation (starting gestation day 8 and through postpartum day 16 via osmotic pump at constant rate $0.25 \mu \mathrm{l} / \mathrm{h}$ at $0,0.25,2.5$ or $25 \mu \mathrm{g} \mathrm{BPA} / \mathrm{kg} / \mathrm{day}$ ) demonstrated altered mammary phenotypes including appearance of alveolar buds and intraductal hyperplasia [41].

In comparison to phthalates and pesticides, there are fewer direct studies demonstrating the associations between human BPA exposure and development of asthma or allergy. However, many studies are suggestive of possible links. BPA exposure appears to influence multiple proinflammatory arms of the immune system. For example, Lee et al. demonstrated in vitro stimulation of keyhole limpet haemocyanin-primed $\mathrm{CD} 4+\mathrm{T}$ cells with BPA significantly enhanced proallergic IL-4 production in a concentrationdependent manner in mice. Both IL-4 and antigen-specific IgE levels were associated with stimulation of $\mathrm{Ca} 2+/$ calcineurin-dependent NF-AT activation [42]. Similarly, Tian et al. demonstrated that ex vivo exposure to BPA resulted in increased production of IL-4 in Th2-dominant mesenteric lymph node cells in mice [43].

Another theme that is becoming evident from the growing literature is that early exposure to BPA is particularly associated with heighted inflammation. For example, prenatal [44] and early postnatal [45] exposure of mice induced greater Th2 polarization (ie greater IL-4, reduced interferon (IFN)- $\gamma$ ). Another important finding in the first paper is that 
prenatal, more so than during adulthood, exposure to BPA was associated with reduced percentages of $\mathrm{T}$ regulatory CD4+CD25+ cells [44], a phenotype associated with atopy [46-48]. Further, the association between prenatal BPA exposure and reduction of $\mathrm{T}$ regulatory cells was found in a related mouse model of oral tolerance and food allergies [49].

However, opposite effects may be observed with low doses of BPA, perhaps related to mouse strain or age. For example, BPA exposure of C57BL/6 strain of mice at age 5 weeks was associated with significant reductions in the Th1 IFN-y production and ConA-stimulated interleukin-10 production [45].

\section{CONCLUSION}

In summary, multiple observational studies have demonstrated an association between exposure to phthalates and the development of asthma and allergies in humans. In vitro and in vivo animal models with different modes of exposure to phthalates support these findings, and provide data suggesting that the association may involve upregulation of allergic immune responses. Associations with exposure to pesticides and the development of respiratory disease, particularly in the pediatric population, are less clear. In particular, there is a lack of prospective studies that focus solely on measured individual pesticides and their role in nonoccupational asthma and allergy development. There is also a dearth of toxicological studies demonstrating the mechanisms by which pesticides exert their effects on the human body. Despite recent evidence suggesting that prenatal or early postnatal exposure to BPA may be deleterious to the developing immune system, even at very low doses, its contribution to asthma pathogenesis, if any, still needs to be elucidated. In animal studies, the doses and blood levels of agents used may not be comparable to actual levels of human exposure, thus translation of results to date to human clinical effects need to be conducted with caution. Currently, there is a lack of studies that utilize biomarkers, such as urinary metabolites, to assess human exposure to these chemicals, independently or in combination.

Even though the three chemicals reviewed in this paper differ in their composition, sources of exposure, and strength of the health effects research, several common themes are apparent. The first is that these chemical exposures are common and the dose level of concern is not known. The second is that prenatal exposure may be a source of particular concern because of potential greater susceptibility of the fetus or because of complex dose response relationships that are not well-understood. The third is that the adverse health effects of these common exposures, especially in regards to asthma and allergies, have not been sufficiently studied.

A key research question is whether or not these ubiquitous chemicals contribute to the development of asthma and allergies in human and, if so, to establish the routes and mechanisms by which they operate. Particular attention should be paid to determining the dose-response relationships and concentrations at which the chemicals become unsafe. Such knowledge could have implications for federal regulation of levels of these chemicals in the environment and in consumer products. A second question is whether early or prenatal exposure to these chemicals is particularly deleterious. Third, how do chemical exposures and genetic makeup interact to heighten susceptibility to chemical exposure on asthma risk?

Cohort-driven epidemiological or translational research, combined with thoughtful experimental design, has great potential to resolve some of these questions. They are particularly relevant topics in this age of "green living," when efforts are being made to minimize the environmental damage caused by common human activities of daily living. Attempts to reduce exposure to chemicals such as phthalates, pesticides, and BPA may not only have environmental repercussions, but may also have public health impact for the developing child.

\section{LIST OF ABBREVIATIONS}

\begin{tabular}{|c|c|c|}
\hline BPA & $=$ & bisphenol A \\
\hline $\mathrm{BBzP}$ & $=$ & n-butyl benzyl phthalate \\
\hline DBuP & $=$ & dibutyl phthalte \\
\hline DEHP & $=$ & di-2-ethyl-hexyl phthalate \\
\hline DEP & $=$ & diethyl phthalate \\
\hline DIBP & $=$ & diisobutyl phthalate \\
\hline DiDP & $=$ & diisodecyl phthalate \\
\hline DIHP & $=$ & diisohexyl phthalate \\
\hline DiNP & $=$ & diisononyl phthalate \\
\hline DnBP & $=$ & di-n-butyl phthalate \\
\hline DOP & $=$ & dioctyl phthalate \\
\hline IL & $=$ & interleukin \\
\hline Ig & $=$ & immunolgobulin \\
\hline IFN & $=$ & interferon \\
\hline $\mathrm{MBuP}$ & $=$ & mono-n-butyl phthalate \\
\hline MEHP & $=$ & mono-(2-ethylhexyl $=$ phthalate \\
\hline MIDP & $=$ & mono-isodecyl phthalate \\
\hline MINP & $=$ & mono-iso-nonyl phthalate \\
\hline $\mathrm{MnBP}$ & $=$ & mono-n-butyl phthalate \\
\hline MOP & $=$ & mono-n-octyl phthalate \\
\hline OVA & $=$ & ovalbumin \\
\hline PBMC & $=$ & peripheral blood mononuclear cells \\
\hline PVC & $=$ & polyvinyl chloride \\
\hline Th & $=$ & T helper \\
\hline
\end{tabular}

\section{CONFLICT OF INTEREST}

The authors have no conflict of interests to declare. Funding source: R01ES014393, P50ES015905.

\section{REFERENCES}

[1] Hoppin J, Umbach D, London S, Alavanja M, Sandler D. Chemical predictors of wheeze among farmer pesticide applicators in the Agricultural Health Study. Am J Respir Crit Care Med 2002; 165: 683-9.

[2] Jaakkola J, Knight T. The role of exposure to phthalates from polyvinyl chloride products in the development of asthma and allergies: 
a systematic review and meta-analysis. Environ Health Perspect 2008; 116; 845-53.

[3] Maffini M, Rubin B, Sonnenschein C, Soto A. Endocrine disruptors and reproductive health: the case of bisphenol-A. Mol Cell Endocrinol 2006; 254-255: 179-86.

[4] Mannino D, Homa D, Akinbami L, Moorman J, Gwynn C, Redd S. Surveillance for asthma--United States, 1980-1999. MMWR Surveill Summ 2002; 51: 1-13.

[5] Salam M, Li Y, Langholz B, Gilliland F. Early-life environmental risk factors for asthma: findings from the Children's Health Study. Environ Health Perspect 2004; 112: 760-5.

[6] NTP-CERHR Monograph on the Potential Human Reproductive and Developmental Effects of Di (Ethylhexyl) Phthalate (DEHP). NTP CERHR MON 2006; 18: i-III76.

[7] Wormuth M, Scheringer M, Vollenweider M, Hungerbuhler K. What are the sources of exposure to eight frequently used phthalic acid esters in Europeans? Risk Anal 2006; 26: 803-24.

[8] Silva M, Barr D, Reidy J, et al. Urinary levels of seven phthalate metabolites in the U.S. population from the National Health and Nutrition Examination Survey (NHANES) 1999-2000. Environ Health Perspect 2004; 112: 331-8.

[9] Rudel R, Camann D, Spengler J, Korn L, Brody J. Phthalates, alkylphenols, pesticides, polybrominated diphenyl ethers, and other endocrine-disrupting compounds in indoor air and dust. Environ Sci Technol 2003; 37: 4543-53.

[10] Adibi J, Whyatt R, Williams P, et al. Characterization of phthalate exposure among pregnant women assessed by repeat air and urine samples. Environ Health Perspect 2008; 116: 467-73.

[11] Frederiksen H, Skakkebaek N, Andersson A. Metabolism of phthalates in humans. Mol Nutr Food Res 2007; 51: 899-911.

[12] Koch H, Muller J, Wittassek M, Angerer J. Influence of alimentary abstinence on body burden to phthalates. Epidemiology 2006; 17: S300.

[13] Fromme H, Bruber L, Schlummer M, et al. Intake of phthalates and di(2-ethylhexyl)adipate: results of the integrated exposure assessment survey based on duplicate diet samples and biomonitoring data. Environ Int 2007; 33: 1012-20.

[14] Clark K, MacKay D, Yamada K. Observed concentrations in the environment. In: Staples C, Ed. The handbook of environmental chemistry, Vol 3, Part Q: Phthalate Esters, Springer: New York, 2003; vol. 3: pp. 125-177.

[15] Jaakkola J, Verkasalo P, Jaakkola N. Plastic wall materials in the home and respiratory health in young children. Am J Public Health 2000; 90: 797-9.

[16] Jaakkola J, Oie L, Nafstad P, Botten G, Samuelsen S, Magnus P. Interior surface materials in the home and the development of bronchial obstruction in young children in Oslo, Norway. Am J Public Health 1999; 89: 188-92.

[17] Bornehag C, Sundell J, Sigsgaard T. Dampness in buildings and health $(\mathrm{DBH})$ : report from an ongoing epidemiological investigation on the association between indoor environmental factors and health effects among children in Sweden. Indoor Air 2004; 14 (Suppl 7): 59-66.

[18] Bornehag C, Sundell J, Weschler C, et al. The association between asthma and allergic symptoms in children and phthalates in house dust: a nested case-control study. Environ Health Perspect 2004; 112: 1393-7.

[19] Kolarik B, Naydenov K, Larsson M, Borehag C, Sundell J. The association between phthalates in dust and allergic diseases among Bulgarian children. Environ Health Perspect 2008; 116: 98-103.

[20] Nakamura R, Teshima R, Sawada J. Effect of dialkyl phthalates on the degranulation and $\mathrm{Ca} 2+$ response of $\mathrm{RBL}-2 \mathrm{H} 3$ mast cells. Immunol Lett 2002; 80: 119-24.

[21] Glue C, Millner A, Bodtger U, Jinquan T, Poulsen L. In vitro effects of monophthalates on cytokine expression in the monocytic cell line THP-1 and in peripheral blood mononuclear cells from allergic and non-allergic donors. Toxicol In Vitro 2002; 16: 657-62.

[22] Glue C, Platzer M, Larsen S, Nielsen G, Skov P, Poulsen L. Phthalates potentiate the response of allergic effector cells. Basic Clin Pharmacol Toxicol 2005; 96: 140-2.

[23] Jepsen K, Abildtrup A, Larsen S. Monophthalates promote IL-6 and IL-8 production in the human epithelial cell line A549. Toxicol In Vitro 2004; 18: 265-9.

[24] Klimisch H, Gamer A, Hellwig J, Kaufmann W, Jackh R. Di-(2ethylhexyl) phthalate: a short-term repeated inhalation toxicity study including fertility assessment. Food Chem Toxicol 1992; 30: 915-9.

[25] Larsen S, Hansen J, Thygesen P, Begtrup M, Poulsen O, Nielsen G. Adjuvant and immuno-suppressive effect of six monophthalates in a subcutaneous injection model with BALB/c mice. Toxicology 2001; 169: 37-51.

[26] Larsen S, Lund R, Nielsen G, Thygesen P, Poulsen O. Adjuvant effect of di-n-butyl-, di-n-octyl-, di-iso-nonyl- and di-iso-decyl phthalate in a subcutaneous injection model using BALB/c mice. Pharmacol Toxicol 2002; 91: 264-72.

[27] Hansen J, Larsen S, Poulsen L, Nielsen G. Adjuvant effects of inhaled mono-2-ethylhexyl phthalate in BALB/cJ mice. Toxicology 2007; 232: 79-88.

[28] Butala J, David R, Gans G, et al. Phthalate treatment does not influence levels of IgE or Th2 cytokines in B6C3F1 mice. Toxicology $2004 ; 201: 77-85$.

[29] Yanagisawa R, Takano H, Inoue K, Koike E, Sadakane K, Ichinose T. Effects of maternal exposure to di-(2-ethylhexyl) phthalate during fetal and/or neonatal periods on atopic dermatitis in male offspring. Environ Health Perspect 2008; 116: 1136-41.

[30] Hoppin J, Umbach D, London S, Alavanja M, Sandler D. Diesel exhaust, solvents, and other occupational exposures as risk factors for wheeze among farmers. Am J Respir Crit Care Med 2004; 169: 1308-13.

[31] Hoppin J, Umbach D, London S, Lynch C, Alavanja M, Sandler D. Pesticides associated with wheeze among commercial pesticide applicators in the agricultural health study. Am J Epidemiol 2006; 163: 1129-37.

[32] Salameh P, Baldi I, Brochard P, Raherison C, Abi S, Salamon R. Respiratory symptoms in children and exposure to pesticides. Eur Respir J 2003; 22: 507-12.

[33] O'Sullivan B, Lafleur J, Fridal K, et al. The effect of pesticide spraying on the rate and severity of ED asthma. Am J Emerg Med 2005; 23: 463-7.

[34] Vial T, Nicolas B, Descotes J. Clinical immunotoxicity of pesticides. J Toxicol Environ Health 1996; 48: 215-29.

[35] Fryer A, Lein P, Howard A, Yost B, Beckles R, Jett D. Mechanisms of organophosphate insecticide-induced airway hyperreactivity. Am J Physiol Lung Cell Mol Physiol 2004; 286: L963-9.

[36] NTP-CERHR Monograph on the Potential Human Reproductive and Developmental Effects of Bisphenol A. NTP CERHR MON 2008; i-III1.

[37] Stahlhut R, Welshons W, Swan W. Bisphenol A data in NHANES suggest longer than expected half-life, substantial nonfood exposure, or both. Environ Health Perspect 2009; 117: 784-9.

[38] Vandenberg L, Hauser R, Marcus M, Olea N, Welshons W. Human exposure to bisphenol A (BPA). Reprod Toxicol 2007; 24: 139-77.

[39] Vandenberg L, Maffini M, Sonnenschein C, Rubin B, Soto A Bisphenol-A and the great divide: a review of controversies in the field of endocrine disruption. Endocr Rev 2009; 30: 75-95.

[40] Tsutsumi O. Assessment of human contamination of estrogenic endocrine-disrupting chemicals and their risk for human reproduction. J Steroid Biochem Mol Biol 2005; 93: 325-30.

[41] Vandenberg L, Maffini M, Schaeberle C, et al. Perinatal exposure to the xenoestrogen bisphenol-A induces mammary intraductal hyperplasias in adult CD-1 mice. Reprod Toxicol 2008; 26: 210-9.

[42] Lee M, Chung S, Kang B, et al. Enhanced interleukin-4 production in CD4+ T cells and elevated immunoglobulin E levels in antigenprimed mice by bisphenol $\mathrm{A}$ and nonylphenol, endocrine disruptors: involvement of nuclear factor-AT and $\mathrm{Ca}^{2+}$. Immunology 2003; 109: 76-86.

[43] Tian X, Takamoto M, Sugane K. Bisphenol A promotes IL-4 production by Th2 cells. Int Arch Allergy Immunol 2003; 132: 240-7.

[44] Yan H, Takamoto M, Sugane K. Exposure to Bisphenol A prenatally or in adulthood promotes $\mathrm{T}(\mathrm{H}) 2$ cytokine production associated with reduction of $\mathrm{CD} 4 \mathrm{CD} 25$ regulatory $\mathrm{T}$ cells. Environ Health Perspect 2008; 116: 514-9.

[45] Sawai C, Anderson K, Walser-Kuntz D. Effect of bisphenol A on murine immune function: modulation of interferon-gamma, IgG2a, and disease symptoms in NZB X NZW F1 mice. Environ Health Perspect 2003; 111: 1883-7.

[46] Francis J, Till S, Durham S. Induction of IL-10+CD4+CD25+ T cells by grass pollen immunotherapy. J Allergy Clin Immunol 2003; 111: 1255-61. 
[47] Karagiannidis C, Akdis M, Holopainen P, et al. Glucocorticoids upregulate FOXP3 expression and regulatory $\mathrm{T}$ cells in asthma. $\mathrm{J}$ Allergy Clin Immunol 2004; 114: 1425-33.

[48] Ling E, Smith T, Nguyen X, et al. Relation of CD4+CD25+ regulatory T-cell suppression of allergen-driven T-cell activation to atopic status and expression of allergic disease. Lancet 2004; 363 : 608-15.

[49] Ohshima Y, Yamada A, Tokuriki S, Yasutomi M, Omata N, Mayumi M. Transmaternal exposure to bisphenol a modulates the development of oral tolerance. Pediatr Res 2007; 62: 60-4.

(C) Kwak et al.; Licensee Bentham Open.

This is an open access article licensed under the terms of the Creative Commons Attribution Non-Commercial License (http://creativecommons.org/licenses/by-nc/3.0/) which permits unrestricted, non-commercial use, distribution and reproduction in any medium, provided the work is properly cited. 